\title{
Analysis of Epileptic and Normal EEG Signals based on Random Walk
}

\author{
Jun MIN ${ }^{1, a}$, Jun WANG ${ }^{2, b^{*}}$ \\ ${ }^{1}$ School of Telecommunications and Information \\ Engineering,Nanjing University of Posts and \\ Telecommunications, Nanjing \\ 210003, Jiangsu,China \\ ${ }^{2}$ School of Geography and Biological Information, \\ Nanjing University of Posts and \\ Telecommunications,Nanjing 210023, \\ Jiangsu,China \\ a1396705817@qq.com, bwangj@njupt.edu.cn \\ *Jun Wang
}

\begin{abstract}
Keywords:random walk, Bayesian inference, epilepsy, EEG signal
Abstract. In this paper, a random walk model was established for the EEG signal. The characteristic parameters qt and at were extracted from the original EEG signal by Bayesian inference method. By comparing the global maximum of the autocorrelation function of the characteristic parameter qt between patients with epilepsy and normal subjects, this paper obtained the following conclusion, the global maximum of the autocorrelation function of the characteristic parameter qt of EEG signal extracted in epilepsy patients is larger than the normal human in the general trend; and the fluctuation of the global maximum of the autocorrelation function in epilepsy patients is also greater, which suggests that the use of random walk model to analyze the EEG signal can be found the difference in patients with epilepsy and normal subjects; therefore, random walk model can be used to analyze the difference between epileptic EEG signal and normal human EEG signals.
\end{abstract}

\section{Introduction}

Epilepsy is a chronic neurological disorders, and it is due to sudden onset of brain neurons abnormal discharge, so that the central nervous system activity is abnormal excitement or inhibition, which has a great impact on the psychological and physical aspects of epilepsy patients. EEG is the basic tool for the diagnosis of epilepsy. It is very important for physiology research and medical application and it is the response of the cerebral cortical or scalp surface brain tissue to the total electrical activity. It contains a lot of brain activity and function information. At present, the common methods of EEG signal processing are: time-frequency analysis[1], artificial neural network[2], nonlinear dynamics[3], independent component analysis[4].

Random walk[5] is an important part of the random process. It is an irregular form of change, in this process of change, every step of the changes are purely random, in our lives, the random walk associated with the natural phenomena, such as the movement of gas molecules, Drops of ink into water, the spread of smell and so on. At present, random walks have been used in many areas, financial analysis, image segmentation and data mining and other aspects.

In this paper, we construct a random walk model[6] for the EEG signal of epilepsy patients and normal people, and use the Bayesian inference[7] to get the characteristic parameter from the EEG signal, and compare the difference between epilepsy patients and normal subjects. The experimental result show that: compare with normal subjects, the global maximum of the autocorrelation function of the characteristic parameter qt is greater, the amplitude of the fluctuation is greater and is more unstable, this show the difference of EEG signal between patient with epilepsy and normal subjects, which suggest that random walk can be used as a method to analyze EEG signal in patients with epilepsy and normal subjects. 


\section{Basic Principle}

\section{Random walk}

Random walk is a mathematical statistical model, known as a stochastic or random process, which is composed of a series of trajectories, each of which is random. It can be used to express irregular changes in the form of random, the core concept of random walk is that the conserved quantity of any random walker corresponds to a law of diffusion, it is similar to the Brownian movement, and it is an ideal mathematical state of Brownian movement. The random walk formula used in this paper is as follows:

$$
u_{t}=q_{t} u_{t-1}+a_{t} n_{t}
$$

In Formula (1), $u_{t}$ is a two-dimensional vector $u_{t}=\left(u_{x t}, u_{y t}\right)$, and in this paper, $u_{x t}$ is the first guide of EEG signal, and $u_{y t}$ is the second guide of EEG signal. $n_{t}$ is also a two-dimensional vector $\mathrm{n}_{\mathrm{t}}=\left(\mathrm{n}_{\mathrm{xt}}, \mathrm{n}_{\mathrm{yt}}\right)$, it is a normal distributed, uncorrelated random noise with unit variance. The Parameter $\left(q_{t}, a_{t}\right)$ represent the characteristics of the two-dimensional vector $u_{t}$. In order to get the parameter $\left(q_{t}, a_{t}\right)$, we use the method of Bayesian inference to get the parameter from $u_{t}$.

\section{Bayesian inference}

Bayesian inference is a method of deducing statistics. This approach uses the Bayesian theorem, and when there is more evidence and information, update the probability of a particular hypothesis, Bayesian inference is one of the most important techniques in statistics. Bayesian updates are particularly important in sequence analysis. Bayesian inference is used in many fields, including science, engineering, medicine, and so on.

In this paper, in order to extract the parameter $q_{t}$ and $a_{t}$ from $u_{t}$ in formula (1), we first get the time-dependent joint probability density $p\left(q_{t+1}, a_{t+1}\right)$ of parameter $q_{t}$ and $a_{t}$ using sequential Bayesian updating, and then get the $\mathrm{q}_{\mathrm{t}}$ and $\mathrm{a}_{\mathrm{t}}$ by calculate the mean of the joint probability density $\mathrm{p}\left(\mathrm{q}_{\mathrm{t}}, \mathrm{a}_{\mathrm{t}}\right)$. we start at time $\mathrm{t}=0$ with a flat prior distribution $\mathrm{p}_{0}(\mathrm{q}, \mathrm{a})$, which can be interpreted as a "first guess" about the parameter values, in this paper, we give the first guess value $p_{0}(q, a)=1 / 40000$. From the measured successive vector $\mathrm{u}_{0}$ and $\mathrm{u}_{1}$, we calculate the likelihood distribution $\mathrm{L}_{1}(\mathrm{q}, \mathrm{a})$, which provides a first information about probable parameter values. And obtain the posterior distribution $\mathrm{p}_{0} \mathrm{~L}_{1}$, and continue on this step, we get the posterior distributions in formula (2):

$$
\mathrm{p}_{\mathrm{t}}=\mathrm{p}_{\mathrm{t}-1} \mathrm{~L}_{\mathrm{t}}
$$

$\mathrm{K}$ is a transformation that both gradual and abrupt parameter changes can be identified. And at last, we perform the same sequential parameter inference in the reverse time direction and combine both distributions. With the iterative multiplication of the formula(2), the posterior distribution would yield an increasingly accurate estimate result.

In formula (2), we use the discretized probability distributions to describe posterior distribution $\mathrm{p}_{\mathrm{t}}$, based on equally spaced parameter values $\mathrm{q}_{\mathrm{i}}, \mathrm{a}_{\mathrm{j}}\left(\mathrm{i} \in\left\{1,2, \ldots, \mathrm{N}_{\mathrm{q}}\right\}, \mathrm{j} \in\left\{1,2, \ldots, \mathrm{N}_{\mathrm{a}}\right)\right.$, a distribution $\mathrm{p}(\mathrm{q}$, a) can be approximated by $\mathrm{N}_{\mathrm{q}} \times \mathrm{N}_{\mathrm{a}}$-dimensional matrix: $(\mathrm{p}(\mathrm{q}, \mathrm{a}))_{\mathrm{ij}}=\mathrm{p}\left(\mathrm{q}=\mathrm{q}_{\mathrm{i}}\right.$, $\left.\mathrm{a}=\mathrm{a}_{\mathrm{j}}\right)$. the multiplication of two distributions is thus reduced to the element-wise multiplication of two matrices, in the paper, we set $\mathrm{N}_{\mathrm{q}}=200$ and $\mathrm{N}_{\mathrm{a}}=200$, so the discretized probability distribution is the $200 \times 200$-dimensional matrix.

As for $L_{t}$ in formula (2), we use the likelihood function, we set $L_{t+1}=p\left(u_{t+1} \mid q_{t+1}, a_{t+1} ; u_{t}\right)$ that describes the probability of observing a certain measurement $u_{t+1}$, given the value of the latent parameters. The likelihood function is given by formula (3):

$$
\mathrm{L}_{\mathrm{t}+1}=\frac{1}{\left(2 \pi \mathrm{a}_{\mathrm{t}+1}{ }^{2}\right)^{\mathrm{d} / 2}} \exp \left(-\frac{\left(\mathrm{u}_{\mathrm{t}+1}-\mathrm{q}_{\mathrm{t}+1} \mathrm{u}_{\mathrm{t}}\right)^{2}}{2 \mathrm{a}_{\mathrm{t}+1}{ }^{2}}\right)
$$


in formula (3), $d$ is the number of the dimension of the $u_{t}(d$ is two in this paper).

\section{Analysis of EEG Signals Based on Random Walking}

\section{Experimental data}

In this paper, the EEG signals of normal subjects and patients with epilepsy were collected from clinical diagnosis by the General hospital of Nanjing Military Region. The recorded length was more than $1 \mathrm{~min}$ and the sampling frequency was $512 \mathrm{~Hz}$. The library records multi-parameter EEG data, including 16 guide signals. EEG data of 21 healthy subjects and EEG data of 21 patients with epilepsy were selected in this paper. And in the experiment, the EEG data of the first guide and the second guide were select for analyzing.

\section{Data preprocessing}

In this paper, we take the first 10000 points of the EEG data, and then divide the EEG data into 10 segment, sub-section followed by [1-1000], [1001-2000], [2001-3000], [3001-4000], [4001-5000], [5001-6000], [6001-7000], [7001-8000], [8001-9000], [9001-10000], and then process each segment of the data.

\section{Experimental process}

In this paper, we firstly construct the random walk model with the formula like equation (1), and for the vector $u_{t}=\left(u_{x t}, u_{y t}\right), u_{x t}$ is the first guide of EEG, $u_{y t}$ is the second guide of the EEG. Then using Bayesian updating to get the characteristic parameter $\left(\mathrm{q}_{\mathrm{t}}, \mathrm{a}_{\mathrm{t}}\right)$. because we divided the EEG data into 10 segments, so we obtain 10 parameter sequences of $\left\{\mathrm{q}_{\mathrm{ti}}, 1 \leq \mathrm{i} \leq 10\right\}$ and $\left\{\mathrm{a}_{\mathrm{ti}}, 1 \leq \mathrm{i} \leq 10\right\}$. In order to decrease the abrupt change of $q_{t}$,we use the method to of average $q_{t}$, firstly, we use the first $q_{t 1}$, calculate the autocorrelation function of $\mathrm{q}_{\mathrm{t} 1}$, and get the global maximum of the autocorrelation function. And then get the mean $\mathrm{q}_{\mathrm{t}}^{\prime}$ of the first three $\left\{\mathrm{q}_{\mathrm{t} 1}, \mathrm{q}_{\mathrm{t} 2}, \mathrm{q}_{\mathrm{t} 3}\right\}$, calculate the autocorrelation function of $\mathrm{q}_{\mathrm{t}}^{\prime}$, and obtain the global maximum of the maximum. third, get the mean $\mathrm{q}_{\mathrm{t}}^{\prime}$ of the five $\left\{\mathrm{q}_{\mathrm{t} 1}, \mathrm{q}_{\mathrm{t} 2}, \mathrm{q}_{\mathrm{t} 3}, \mathrm{q}_{\mathrm{t} 4}, \mathrm{q}_{\mathrm{t} 5}\right\}$, calculate the autocorrelation function of $\mathrm{q}_{\mathrm{t}}^{\prime}$, and obtain the global maximum of the autocorrelation. Finally, using the ten result of $q_{t}$, get the means $q_{t}^{\prime}$ of the $q_{t}$, and calculate the global maximum of the autocorrelation function of $\mathrm{q}_{\mathrm{t}}^{\prime}$. From this four result, We can find more and more accurate experimental results.

\section{Experimental results and analysis}

Using the above four steps of calculation, we calculate the result of all EEG data, which contains 21 normal subjects and 21 patients with epilepsy. And compare the maximum of their autocorrelation functions of $q_{t}^{\prime}$. the experimental results are shown in Figure 1- Figure 4.

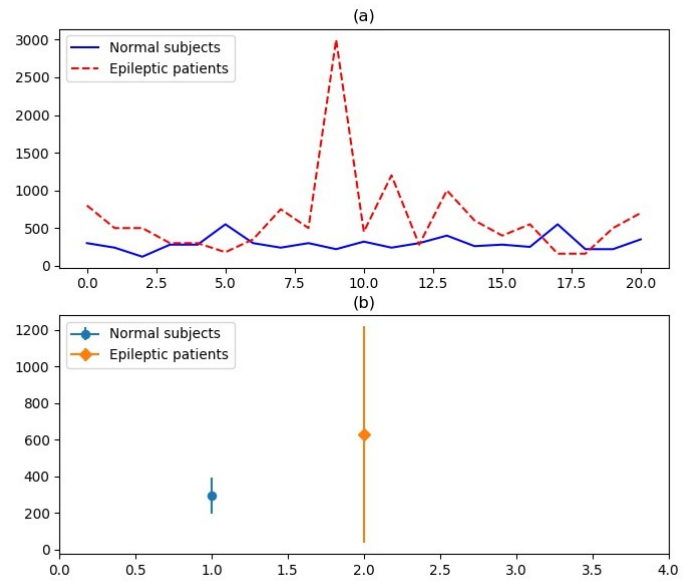

Fig. 1. Use the first $\mathrm{q}_{\mathrm{t}}$, calculate global maximum of the autocorrelation function of $\mathrm{q}_{\mathrm{t}}$. 

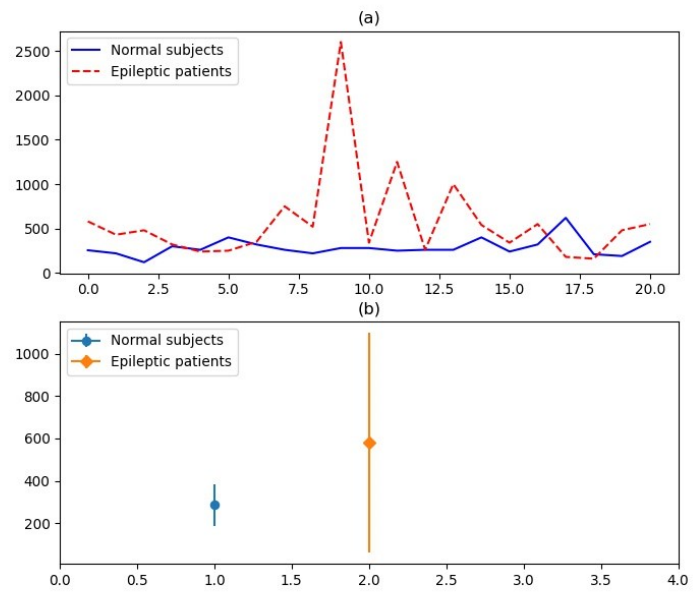

Fig. 2. Use the first three $q_{t}$, get the mean $q_{t}^{\prime}$ and calculate global maximum of the autocorrelation function of $q_{t}^{\prime}$.
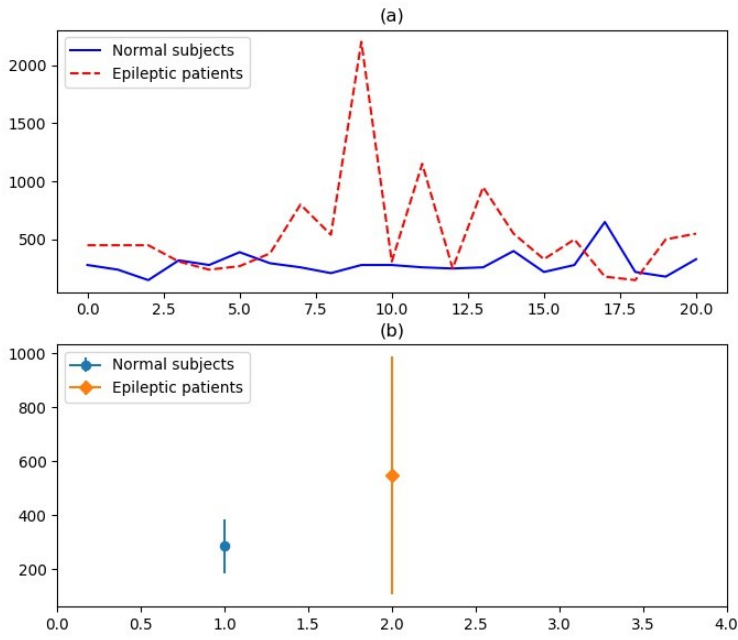

Fig. 3. Use the first five $q_{t}$, get the mean $q_{t}^{\prime}$ and calculate global maximum of the autocorrelation function of $\mathrm{q}_{\mathrm{t}}^{\prime}$.
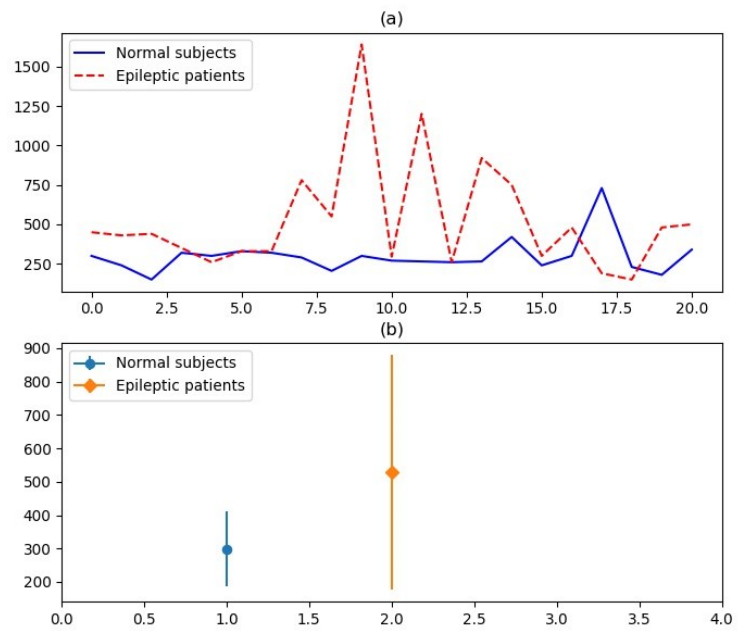

Fig. 4. Use the first ten $\mathrm{q}_{\mathrm{t}}$, get the mean $\mathrm{q}_{\mathrm{t}}^{\prime}$ and calculate global maximum of the autocorrelation function of $\mathrm{q}_{\mathrm{t}}^{\prime}$. 
From Fig1 to Fig(4), the subgraph (a) represent the comparison of autocorrelation function of $\mathrm{q}_{\mathrm{t}}^{\prime}$ between normal subject and patient with epilepsy. And the subgraph (b) is the mean and standard deviation of the autocorrelation fuction of $\mathrm{q}_{\mathrm{t}}^{\prime}$.

From Fig1(a) - Fig4(a), we can see that in the general trend, the global maximum of the autocorrelation function of $\mathrm{q}_{\mathrm{t}}^{\prime}$ in epilepsy patients is always larger than that of the normal person, whether or not average the $\mathrm{q}_{\mathrm{t}}$, although the method of average decrease the maximum of autocorrelation function of $\mathrm{q}_{\mathrm{t}}^{\prime}$. and we can also see that the maximal autocorrelation function of $\mathrm{q}_{\mathrm{t}}$ in patient with epilepsy is more volatile and more unstable. This show that the patient with epilepsy have more unstable EEG signals.

In order to show the above conclusion clearly, we draw the mean square deviation graph from fig 1(b) - fig4(b). in fig1(b) - fig4(b), we can see that the mean square deviation graph of normal people range from $200-400$. But the mean square deviation graph of patient with epilepsy is changing, with the increasing in the number of $q_{t}$, the range of mean square deviation graph is decreasing, when average first ten $\mathrm{q}_{t}$, the range of epilepsy patient is about $150-1000$, this completely cover the range of normal people. And this picture also show the means of the maximum value of the $q_{t}^{\prime}$ autocorrelation function. The normal people is about 300, but the epilepsy is about 550 .

According to fig1-fig4, we can conclude that, for the global maximum of the autocorrelation function of $q_{t}^{\prime}$, the value of normal subjects is fluctuate near 300 , and the volatility is not very large, but the patients is near 550, and the volatility is very large, its fluctuation range contains the range of normal people's fluctuation range. This reflects the instability of epileptic signals on the side, we deduce that the patient with epilepsy can get high value of the global maximum of the autocorrelation function of $\mathrm{q}_{\mathrm{t}}^{\prime}$ is due to the abnormal discharge of brain.

\section{Conclusion}

In this paper, we construct a random walk model to analyze EEG signal, and use the Bayesian inference to extract the characteristic parameter of EEG signal, and obtain the characteristic parameter $\left(q_{t}, a_{t}\right)$, and calculate the autocorrelation function of $q_{t}^{\prime}$. by comparing the global maximum of autocorrelation function of $q_{t}^{\prime}$ between normal subjects and patients with epilepsy, we find that in general trend, for the global maximum of autocorrelation function of $q_{t}^{\prime}$, the value in patients with epilepsy is higher than normal people, and the fluctuation in normal is more stable, but the patient with epilepsy is not stable, it contains all the scope which the normal people covers. This result reflect the instability of EEG in epileptic patient. The experimental result show that random walk model can be used to analyze the EEG signal and find the difference between normal people and patient with epilepsy.

\section{Acknowledgement}

Project supported by the project is supported by the National Natural Science Foundation of China (Grant Nos. 61271082, 31671006), Jiangsu Provincial Key R \& D Program (Social Development) (Grant No.BE2015700, BE2016773), the Natural Science Foundation of Jiangsu Province (Grant No. BK20141432),Natural Science Research Major Program in Universities of Jiangsu Province (Grant No.16KJA310002).

\section{References}

[1] Dliou A, Latif R, Laaboubi M. Arrhythmia ECG signal analysis using non parametric time-frequency techniques[J]. International Journal of Computer Applications, 2012, 41(4).

[2] Reference to a chapter in an edited book: Rai H M, Trivedi A, Shukla S. ECG signal processing for abnormalities detection using multi-resolution wavelet transform and Artificial Neural Network classifier[J]. Measurement, 2013, 46(9): 3238-3246. 
[3] Owis M I, Abou-Zied A H, Youssef A B M. Study of features based on nonlinear dynamical modeling in ECG arrhythmia detection and classification[J]. IEEE transactions on Biomedical Engineering, 2002, 49(7): 733-736.

[4] Iriarte J, Urrestarazu E, Valencia M. Independent component analysis as a tool to eliminate artifacts in EEG: a quantitative study[J]. Journal of clinical neurophysiology, 2003, 20(4): 249-257.

[5] Spitzer F. Principles of random walk[M]. Springer Science \& Business Media, 2013.

[6] Metzner C, Mark C, Steinwachs J, et al. Superstatistical analysis and modelling of heterogeneous random walks.[J]. Nature Communications, 2015, 6:7516.

[7] Box G E P, Tiao G C. Bayesian Inference in Statistical Analysis[M]// Bayesian inference in statistical analysis /. Wiley, 1992:478-479. 